\title{
5. Tanstuzumab Treatment for Breast Cancer
}

\author{
Kiyohiko Hatake, Nahomi Tokudome and Yoshinori Ito
}

Key words: molecular target therapy, tanstuzumab, breast cancer

(DOI: 10.2169/internalmedicine.46.1783)

\section{Introduction}

Molecular target therapy has been introduced and it has been successfully approved for use in Japan. Compared to USA and EU, it took a few more years to obtain approval from the Ministry of Welfare, Health, and Labor. Breast cancer, in particular metastatic or relapsed breast cancer was treated by chemotherapy or radiation to improve the quality of life or to prolong survival. Her-2, is a member of the human epidermal growth factor receptor family, and its ligand has not been identified. Positive cases of HER2 showed a poor prognosis and were chemotherapy resistant. Before the introduction of the results of adjuvant therapy using CAF (cyclophosphamide, adriamycin, and 5-fluorouracil) or FEC (5-fluorouracil, epirubicin, and cyclophosphamide), the use of anthracyclines was not recognized. The use of anthracyclines gave rise to the improvement of adjuvant chemotherapy. Trastuzumab, improved the poor prognostic group with metastatic or relapsed breast cancer patients. In Japan, approximately 3,000 Her-2 positive patients receive trastuzumab alone or in combination with taxane or capecitabine. The presence of Her-2 is as important as the peroxidase reaction in acute leukemia, and causative infectious bacteria in pneumonia. Her-2 is so important because it provides different therapeutic strategies for metastatic breast cancer.

First of all, laboratory establishment of HER-2 status is an important step to determine whether the patient's tissue is HER-2 positive or negative. Pathological examination should be done before determining the treatment protocol. The treatment goal is absolutely cure, but in relapsed cases after an adjuvant setting or in metastatic disease of lifethreatening organs the treatment is changed to care or prolorging life.

\section{Basic Aspects of Her-2}

Breast cancer is generally located in the lateral upper portion of both breasts. The risk factors as follows; age $>40$ years old, not married, first delivery over 30 years old, menarche older than 11 years old, menopause over 55 years old, obesity over 1:2, higher fat, higher nutrition, alcohol drinking, and presence of family history of breast cancer. America has a greater incidence of breast cancer, than Japan, however the sncidence has been increasing gradually. Here, there are 35,000 patients/year with breast cancer including 3,500 stage IIIb/IV patients/year and 3,200 unresectable patients/year. Of these patients, there are 9,500 relapsed patients/year, and $25 \%$ of the patients show the presence of Her-2. The 3,150 annual Her-2 positive patients should be indicated for trastuzumab-containing treatment. As for treatment strategies, we have neoadjuvant chemotherapy for patients before surgical operation, and just after operation we have adjuvant therapy and chemotherapy for relapsed patients or metastatic patients.

Her-2 is an epidermal growth factor receptor (EGFR) located in the cell membrane of epithelial cells and cancer cells. The extracellular domain has 632 amino acids, and the transmembrane domain, 22 amino acids, and the intracellular domain, 580 amino acids. Trastuzumab binds to the extracellular domain of EGFR, and therefore blocks signal transduction to the nucleus, and inhibits gene activation.

Trastuzumab is a chimeric monoclonal antibody that is biotechnologically fused with human antibody gene. In 1987, Her-2 was identified as a poor prognostic factor, and was targeted for cancer therapy. In 1981, murine Her-2/neu was cloned and in 1985, human Her-2/neu was cloned. In 1990, murine monoclonal antibody 4D5 against human Her2 was produced and in 1992 a phase I trial using recombinant human monoclonal antibody was conducted; phase II was conducted in 1993, and phase III was in 1995. In 1998, trastuzumab was approved in U.S.A. and in 2000 in EU. In Japan, a phase I trial was conducted in 1996, and it was approved for metastatic breast cancer in 2001. Trastuzumab is an IgG (1) humanized antibody that has a molecular weight of $148 \mathrm{kD}$ with high affinity $(\mathrm{Kd}=0.1 \mathrm{nM})$, and demonstrates the activity of antibody-dependent cytotoxicity (ADCC) via NK cells or monocytes. Trastuzumab also inhibits dimerization of EGFR after binding to EGFR. Down regulation of Her-2 and signal transduction of Her-2 is inhibited. Cell cy- 
cle of breast cancer cells is arrested via p21Kip, and angiogenesis is inhibited with chemosensitization to chemotherapeutic drugs. In a phase I trial, also in Japan, the safety and toxicity of this drug was examined. The initial dose is usually $4 \mathrm{mg} / \mathrm{kg}$ followed by $2 \mathrm{mg} / \mathrm{kg}$ administered weekly. Her-2 3+ or FISH+ in Her-2 2+ patients should be administered as an indication. If the patients under went biopsy or operation, the presence or absence of Her-2 should be examined by FISH or IHC (immunohistochemistry). For the standardization of a positive test of Her-2, Dako Hercep Test is the standard test; Path Vysion FISH assay is for FISH. In the first line treatment for metastatic breast cancer in IHC $3+$ cases, the response rate was $35 \%$, and its define please was 3.5 months, and the overall survival was 24.4 months. In the second/third line, the response rate was $18 \%$, and TTP was 3.3 months. Combination chemotherapy was better than monotherapy, especially with taxanes, including paclitaxel or docetaxel. In the first line setting, paclitaxel plus trastuzumab gave a response rate of $49 \%$, and response duration was 7.1 months, compared to paclitaxel alone where the response rate was $17 \%$, and duration was 3.0 months. Docetaxel plus trastuzumab demonstrated a response rate of $61 \%$, duration was 10.6 months, compared to docetaxel alone which induced a response rate of $34 \%$ and duration of 6.1 months. The addition of trastuzumab improved both the response rate and survival. Adverse events were reported as follows: fever $(32.1 \%)$, chilliness $(12.1 \%)$, nausea $(7.2 \%)$, vomiting (6.5\%), leukocytopenia (5.4\%), dyspnea (3.7\%), and asthenia (2.3\%). Severe adverse events including those over grade 3 showed leukocytopenia (2.5\%), dyspnea $(1.4 \%)$, and neutropenia $(1.1 \%)$. Infusion reaction is a specific adverse event for monoclonal antibody therapy. To prevent infusion reaction, premedication should be given 30 min before the administration of trastuzumab. Cardiac dysfunction after the use of trastuzumab was reported, especially after the use of anthracyclines. Cardiologists should be regularly consulted before and after the use of trastuzumab for the examination of ultrasonography or scintigram. Most severe adverse events, including infusion reaction, occurred with the first administration.

Medical cost is an important matter. Financial consideration is very important for the patient. Trastuzumab costs 3,000 US $\$$ for one month. In the presence of Her-2 showed a high risk in endocrine responsive cases.

\section{Recent Development}

Neoadjuvant therapy with transtuzumab, paclitaxel, and epirubicin significantly improved the pathological complete remission rate in Her-2 positive operable breast cancer.

In Japan, a prospective randomized trial with adjuvant chemotherapy or neoadjuvant therapy in Her-2 3+ cases should be performed. Standard treatment including trastuzumab should be into the local area.

\section{References}

1. Buzdar AU, Ibrahim NK, Francis D, et al. Significantly higher pathologic complete remission rate after neoadjuvant therapy with transtuzumab, paclitaxel, and epirubicin chemotherapy: Results of a randomized trial in human epidermal growth factor receptor 2positive operable breast cancer. J Clin Oncol 23: 3676-3685, 2005 .

(C) 2007 The Japanese Society of Internal Medicine http://www.naika.or.jp/imindex.html 